\title{
PAPER
}

\section{Previous sound effects on loudness in paired comparison experiments}

\author{
Junji Yoshida ${ }^{1, *}$, Hiroshi Hasegawa ${ }^{2, \dagger}$ and Masao Kasuga ${ }^{1}$ \\ ${ }^{1}$ Graduate School of Engineering, Utsunomiya University, \\ 7-1-2 Yoto, Utsunomiya, 321-8585 Japan \\ ${ }^{2}$ Department of Information Science, Utsunomiya University, \\ 7-1-2 Yoto, Utsunomiya, 321-8585 Japan
}

(Received 14 June 2005, Accepted for publication 24 November 2005)

\begin{abstract}
In this study, we investigated the effects of a previous sound on loudness by performing paired comparison experiments. Two pure tones measuring 74 to $86 \mathrm{~dB} \mathrm{SPL}$ at $500 \mathrm{~Hz}$ were presented monaurally at a certain interstimulus interval (ISI), which was set between 0.5 and $8 \mathrm{~s}$. One of the pure tones was presented in one ear, and then the other was presented in the contralateral or ipsilateral ear. The subject compared the loudness of two pure tones and responded which sound was felt louder. The effect of presentation order in the paired comparison was calculated from the experimental results, and then the previous sound effect was obtained from the presentation order effect. As results, the sound presented second in the ipsilateral ear was perceived to be about $1 \mathrm{~dB}$ softer than the first sound at an ISI of $0.5 \mathrm{~s}$ even when both sounds had the same SPL. On the contrary, the second sound in the contralateral ear was perceived to be about $1 \mathrm{~dB}$ louder than the first sound significantly. This effect is referred to as "auditory reinforcement." The effect level of auditory reinforcement decreased as ISI increased.
\end{abstract}

Keywords: Auditory reinforcement, Previous sound effect, Contralateral ear

PACS number: 43.66.Mk, 43.66.Ed [DOI: 10.1250/ast.27.147]

\section{INTRODUCTION}

The loudness of a sound is affected by other sounds heard previously. There are many studies of the effects of a previous sound [1,2], including the effects of auditory fatigue [3-6], loudness recalibration [7-12], and loudness enhancement [13-16]. Most of these phenomena involve the deterioration of the loudness of a sound by perceiving a previous sound, with the exception of loudness enhancement. Auditory fatigue is a phenomenon in which the absolute threshold of hearing decreases after hearing a loud sound [3-6]. Loudness recalibration, which is sometimes called ILR (induced loudness reduction), is a phenomenon whereby a sound heard hundreds of milliseconds after a loud sound is perceived to be softer than the same sound perceived without the loud sound [7-12]. Loudness enhancement is the only phenomenon in which the loudness of a sound appears to be increased by perceiving a sound previously [13-16]. Also, the relationships among these phenomena have been discussed in various studies $[11,17,18]$, and it was generally believed that loudness

\footnotetext{
*e-mail: yoshida@vibra.is.utsunomiya-u.ac.jp

†e-mail: hasegawa@is.utsunomiya-u.ac.jp
}

enhancement is closely associated with loudness recalibration. Scharf et al. mentioned that the observed enhancement is a kind of loudness recalibration based on a reconsideration of the experimental method in the loudness enhancement study of Elmasian et al. [17]. In addition, Marks et al. performed experimental demonstrations of both phenomena (recalibration and enhancement) and concluded that there is no actual effect of increasing loudness in the experiment by Elmasian et al. [11].

In the recalibration experiment of Marks et al. and the enhancement experiment of Elmasian et al., three pure tones were used: the previous sound (for enhancement or recalibration), the target sound (to be enhanced or recalibrated), and the comparison sound (which was presented after the target sound for measuring the loudness of the target sound). In these experiments, they carried out two trials. In the first trial, the loudness of the target sound was determined by the subject by adjusting the sound pressure level (SPL) of the comparison sound in the case without the previous sound. In the second trial, the loudness of the target sound was measured in the case with the previous sound. After presenting the previous sound, the effect level was then calculated as the difference between the measured loudnesses from the conditions with and without the 
previous sound. In the recalibration experiment, a sound whose frequency was different from those of the previous and target sounds was used as the comparison sound to prevent the influence of the previous sound on the comparison sound $[9,17]$. As a result, it was found that the measured loudness of the target sound was decreased by presenting the previous sound [7-12]. On the other hand, in the enhancement experiment, the frequencies of all the sounds (previous, target, and comparison sounds) were the same, and it was obtained that the measured loudness of the target sound was increased by presenting the previous sound [13-16]. From the difference between the above experimental conditions, Scharf and Marks et al. considered that the effect of increasing loudness in the enhancement experiment is a pseudophenomenon caused by the influence of the previous sound on the comparison sound [17], and Marks et al. performed experimental demonstration of the phenomenon [11].

For the above-mentioned reason, the phenomenon of increasing loudness in the enhancement experiment by Elmasian et al. is generally not recognized as the actual effect at present. Also, there are few other studies of the verification as to whether there is an increasing effect of loudness in sequential sound effects.

In this study, the clarification of an increasing effect of loudness towards sequential sounds is attempted in detail by performing comparison experiments using paired sounds at various interstimulus intervals (ISIs) and by presenting one of the paired sounds in one ear followed by the second sound in the ipsilateral or contralateral ear. Two pure tones are used in these experiments, which differs from the case in which three sounds are used in the recalibration and enhancement experiments, to prevent the recalibration effect on the third (comparison) sound in the evaluation of the loudness of the target sound. The effects of the previous sound are evaluated as the effects of presentation order in the paired comparison experiments in this study. The presentation order effects are calculated as a function of ISI from the response probability transitions in the experiments. In addition, the SPL dependencies of the previous sound effects are investigated by changing the SPL of the employed sounds.

\section{EXPERIMENT 1}

Paired comparison tests were performed using two pure tones to evaluate the effect of presentation order.

\subsection{Apparatus and Stimulus}

Two pure tones were used as experimental stimuli, both having a frequency of $500 \mathrm{~Hz}$. Presenting a pair of tones at the same frequency and at the same duration is important in obtaining the effect of presentation order (previous sound effect) in this experimental method. Also, it could be assumed that the effect is not obtained significantly when the duration of the first sound is considerably short. On the other hand, auditory adaptation might occur when the duration is too long [1,2]. Hence, the duration of the first sound was set at $5 \mathrm{~s}$, and the same duration of $5 \mathrm{~s}$ was set for the second sound, that was relatively longer than those of previous studies using three pure tones [8,11,14]. A sound stimulus at SPL of $80 \mathrm{~dB}$ was used as the base sound. Sound stimuli at SPLs of 74, 77, 78, 79, 80, 81, 82, 83, and $86 \mathrm{~dB}$ were compared with the base sound stimulus. These SPLs were calibrated using an artificial head microphone (Head Acoustics HMS 3). The interval of SPLs was set at $1 \mathrm{~dB}$ from 77 to $83 \mathrm{~dB}$ SPLs to obtain a distribution of the evaluation. The stimuli at 74 and $86 \mathrm{~dB}$ SPLs, where most people can detect the difference in loudness compared with that of the base sound ( $80 \mathrm{~dB}$ SPL) [19], were used to confirm the hearing acuity of the subjects. The ISIs between the first and second sounds were set at $0.5,1,2$, 4 , and $8 \mathrm{~s}$. All pairs of the base and comparison sound stimuli were recorded on digital audiotapes at a sampling frequency of $44.1 \mathrm{kHz}$ and presented to the subjects monaurally via headphones (Sony closed-type headphones, MDR-Z600).

\subsection{Subjects}

Eleven males (21 to 24 years) and two females (22 years) participated in this experiment. All the subjects had normal hearing acuity.

\subsection{Procedure}

This experimental procedure was almost the same as the method of evaluating the loudness difference limen [19]. The first of a comparison pair of sounds was presented in one ear and the second was presented in the contralateral or ipsilateral ear. The ISIs of the comparison pairs were set at $0.5,1,2,4$, and $8 \mathrm{~s}$. The comparison tests were performed for each ISI. There are, therefore, five sessions in total. The comparison pairs had two presentation orders, in which the base sound stimulus was presented either first or second, and two presentation types, in which either the second stimulus was presented in the contralateral or ipsilateral ear. Thus, each comparison pair had four general trial types. In all, there were 36 different trial conditions ( 9 comparison sound stimuli $\times 4$ general trial types) per ISI. A total of 468 trials (36 conditions $\times 13$ subjects) were conducted for each ISI. In addition, to offset the influence of the difference in sensitivity between the left and right ears, the number of presentation trials was adjusted, in which the first stimulus was presented to the left ear and the second stimulus was presented to the right ear and vice versa. The obtained results were then analyzed together. Each session took between 10 and 15 minutes. After the presentation of each comparison pair, the subjects 
were asked to respond to the following question on the answer sheet:

Which sound was louder?

(a) The first sound was louder

(b) Both sounds were equally loud

(c) The second sound was louder

\subsection{Analysis}

In this study, the experimental conditions were almost the same as those for a standard experimental method regarding the loudness difference limen [19]. On the basis of this, the presentation order effect was calculated according to the calculation method for difference limens taking into account the presentation order effect [19-22]. The order effect was calculated by fitting Gaussian distributions to the response probabilities of the comparison evaluations at each ISI and each presentation type (the second sound stimulus was presented in the contralateral or ipsilateral ear). To demonstrate the validity of this fitting method, an experimental data set obtained at an ISI of $0.5 \mathrm{~s}$ for the ipsilateral ear was used.

First, the probability of each answer ((a), (b), or (c) in Sect. 2.3) was calculated for the case in which the base sound stimulus was presented first and for the case in which the base sound stimulus was presented second. Figure 1 shows the calculated probabilities for the experiments in which the base sound stimulus was presented first. When $P_{a} \square$ (The base sound was perceived as being louder

$P b$ The sounds were perceived to be equally loud

$P_{c}$ The comparison sound was perceived as being louder (The second sound was louder)

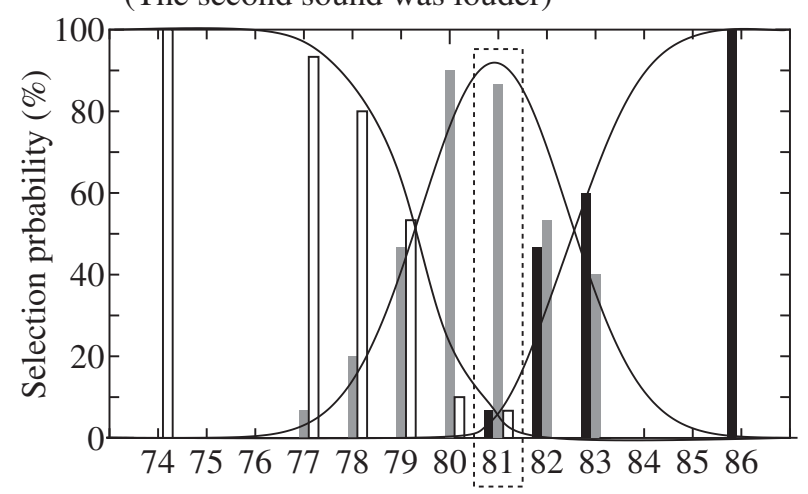

Sound pressure level of the comparison sound $(\mathrm{dB})$

Fig. 1 Selection probabilities when the base sound was presented before the comparison sound at an ISI of $0.5 \mathrm{~s}$ in the ipsilateral ear. The white, gray, and black bars indicate the probabilities of the following responses: "The first (base) sound was louder," "Both sounds were equally loud," and "The second (comparison) sound was louder," respectively. The solid lines show the Gaussian distribution curves fitted to the response probabilities. the first stimulus was $80 \mathrm{~dB}$ SPL (base sound) and the second stimulus was $81 \mathrm{~dB}$ SPL (comparison sound), the obtained probabilities $P_{\mathrm{a}}, P_{\mathrm{b}}$, and $P_{\mathrm{c}}$ were $6.7,86.7$, and $6.7 \%$, respectively, as shown in the area surrounded by the dotted line. $P_{\mathrm{a}}, P_{\mathrm{b}}$, and $P_{\mathrm{c}}$ indicate the probabilities of the following responses: "The first sound was louder," "Both sounds were equally loud," and "The second sound was louder," respectively. The subjective loudness values of the base sound at $80 \mathrm{~dB}$ SPL and the comparison sound at $81 \mathrm{~dB}$ SPL were assumed to vary according to the Gaussian distributions $\phi\left(x_{80} ; 80, \sigma^{2}\right)$ and $\phi\left(x_{81} ; 81, \sigma^{2}\right)$, respectively. $x_{80}$ and $x_{81}$ indicate the subjective loudness values, where the means are 80 and 81 , respectively, and $\sigma$ is the standard deviation of the distributions. Then, the distribution of responses in the comparison of these sound stimuli depends on $\phi\left(x_{81-80} ; 81-80,2 \sigma^{2}\right)$. Figure 2 shows the probability distribution of the paired comparisons obtained with the three possible responses. In this figure, $-C$ and $C$ indicate the cut-off points at which the responses change from "softer" to "the same" and from "the same" to "louder", respectively. When the second sound stimulus is perceived as being $\alpha \mathrm{dB}$ softer than the first sound stimulus depending on the presentation order, the distribution becomes $\phi\left(x ;(81-\alpha)-80,2 \sigma^{2}\right)$. By adopting this distribution, $P_{\mathrm{a}}$, $P_{\mathrm{b}}$, and $P_{\mathrm{c}}$ can be represented as

$$
\begin{aligned}
& P_{\mathrm{a}}=\int_{-\infty}^{-C} \phi\left(x ;(81-\alpha)-80,2 \sigma^{2}\right) d x, \\
& P_{\mathrm{b}}=\int_{-C}^{C} \phi\left(x ;(81-\alpha)-80,2 \sigma^{2}\right) d x, \\
& P_{\mathrm{c}}=\int_{C}^{\infty} \phi\left(x ;(81-\alpha)-80,2 \sigma^{2}\right) d x .
\end{aligned}
$$

On the other hand, when the first stimulus is $81 \mathrm{~dB}$ SPL (comparison sound) and the second stimulus is $80 \mathrm{~dB}$ SPL (base sound), the distribution depends on $\phi(x ; 81-(80-$

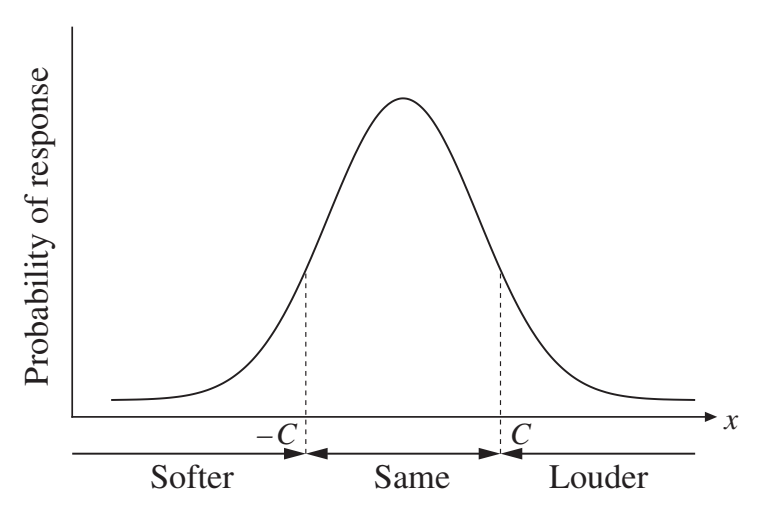

Fig. 2 Response probability distribution of paired comparison. Here, $x$ represents any subjective value and $C$ and $-C$ indicate the cut-off points at which the responses change from "the same" to "louder" and from "softer" to "the same," respectively. 
$\alpha), 2 \sigma^{2}$ ). Also, it was assumed that the order effect $\alpha$ and the cut-off points $\pm C$ are constant for all of the comparison pairs employed in this experiment because the SPL differences between the first and second stimuli $(0$ to $\pm 6 \mathrm{~dB}$ ) are sufficiently small compared with the $80 \mathrm{~dB}$ SPL (base sound).

By applying the above method for each comparison pair, the residual sum of squares (RSS) for all responses between the measured and calculated probabilities from the distribution can be expressed as a function of $C, \sigma$, and $\alpha$ at each ISI and presentation type. $C, \sigma$, and $\alpha$ that give the smallest RSS were then calculated, and the most suitable distribution was constructed. The solid curves in Fig. 1 show the constructed distributions for $P_{\mathrm{a}}, P_{\mathrm{b}}$, and $P_{\mathrm{c}}$. From this, $\alpha$ was obtained as the effect of presentation order. The presentation order effect $\alpha$ indicates that the second sound is perceived to be $\alpha \mathrm{dB}$ louder than the first sound while both sounds have the same SPL, i.e., it indicates that the order effect level to the loudness of the second sound by perceiving the previous sound (first sound). Therefore, the calculated presentation order effect could be regarded as the previous sound effect in the comparison experiment. By using the calculated standard deviation $\sigma$ of the distribution and a sample size equal to the number of comparisons, a 95\% confidence interval was calculated for the previous sound effect. This analysis was carried out for both the contralateral and ipsilateral ears. The influence of the difference in sensitivity between the left and right ears is eliminated from the calculated presentation order effect by offsetting the influence under the experimental conditions in Sect. 2.3. The above procedure was performed for each ISI.

\subsection{Results}

The previous sound effect in the contralateral and ipsilateral ears for each ISI are shown in Fig. 3. The solid and empty circles indicate the effects in the contralateral and ipsilateral ears, respectively. The error bars show the 95\% confidence intervals. In the contralateral ear, the subjects perceived the second sound stimulus to be approximately $1 \mathrm{~dB}$ louder than the first sound even when both sounds had the same SPL at an ISI of 0.5 to $1 \mathrm{~s}$. In contrast, in the ipsilateral ear, the subjects perceived the second sound stimulus to be approximately $1 \mathrm{~dB}$ softer than the first sound at an ISI of 0.5 to $2 \mathrm{~s}$. This result for the ipsilateral ear is similar to that of the previous study by Yost et al. [9], in which they verified a loudness recalibration level under conditions that involved recalibrating and target sound stimuli with the same SPL. The order effect in both the contralateral and ipsilateral ears became less noticeable as ISI was increased. From these results, it was found that the previous sound effect in the ipsilateral ear has a feature for decreasing the loudness of the second presented sound,

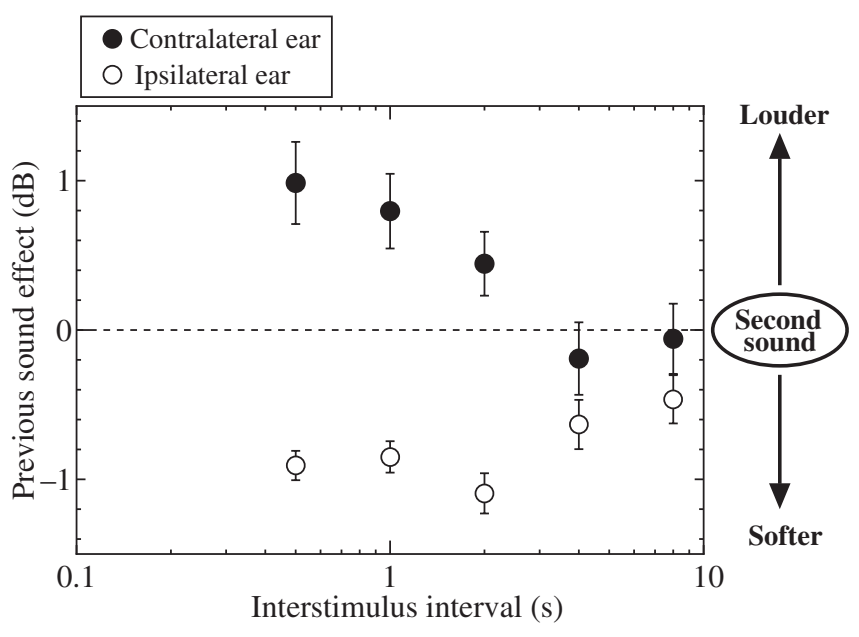

Fig. 3 Previous sound effect in the contralateral and ipsilateral ears when the base sound is $80 \mathrm{~dB}$ SPL. The solid and empty circles indicate the effects in the contralateral and ipsilateral ears, respectively. The error bars show the $95 \%$ confidence intervals. When the order effect is positive, the second sound is perceived to be louder. In contrast, the second sound is perceived to be softer when the effect is negative.

and the effect in the contralateral ear has a feature to increase the loudness of the second sound. These previous sound effects were suppressed as ISI increased. Hereinafter, this increasing effect of loudness in the contralateral ear is referred to as "auditory reinforcement." Furthermore, the confidence intervals show that auditory reinforcement was significant for ISIs of less than $2 \mathrm{~s}$ when the SPL of the base sound stimulus was $80 \mathrm{~dB}$. In the ipsilateral ear, auditory sensitivity deteriorated significantly for ISIs of less than $2 \mathrm{~s}$.

\section{EXPERIMENT 2}

In Experiment 1, it was found that loudness was increased by approximately $1 \mathrm{~dB}$ by the previous sound effect in the contralateral ear (auditory reinforcement) with an approximately $80 \mathrm{~dB}$ SPL sound. In this experiment, we attempted to clarify the dependence of auditory reinforcement on the SPL of the previous sound. To accomplish this, an experiment in which the SPLs of sound stimuli were varied was carried out.

\subsection{Apparatus, Stimulus, and Procedure}

The experimental apparatus and procedure were identical to those in Experiment 1 except for the SPLs. A signal having SPL of $60 \mathrm{~dB}$ was used as the base sound stimulus. The SPLs of the comparison sound stimuli were 54, 57, 58, $59,60,61,62,63$, and $66 \mathrm{~dB}$.

\subsection{Subjects}

Fourteen males (21 to 24 years) and two females (22 years) participated in this experiment. All the subjects had normal hearing acuity. 


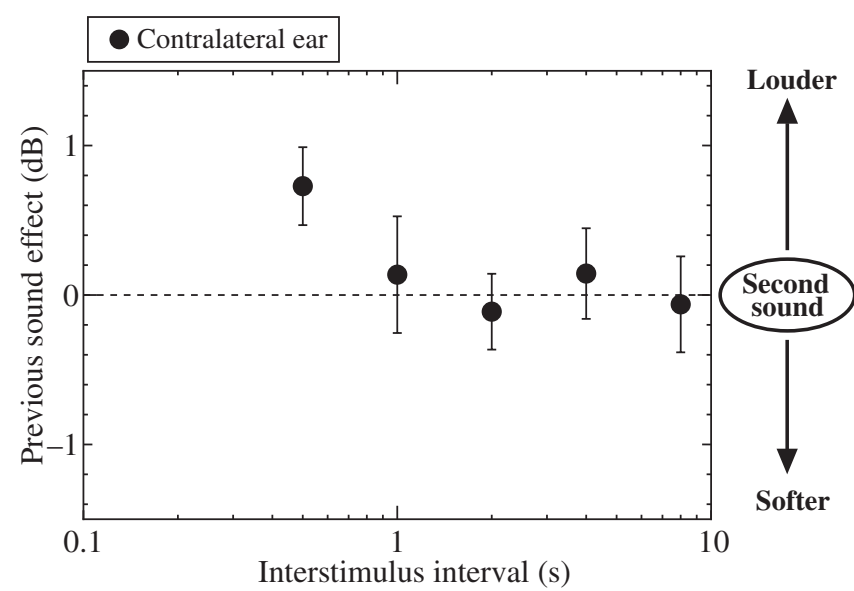

Fig. 4 Previous sound effect in contralateral ear when base sound is $60 \mathrm{~dB}$ SPL. The solid circles indicate the effects in the contralateral ear. The error bars show the $95 \%$ confidence intervals.

\subsection{Results}

Figure 4 shows the previous sound effect in the contralateral ear for each ISI when the SPL of the base sound stimulus was $60 \mathrm{~dB}$. The solid circles indicate the effects in the contralateral ear. The error bars indicate the 95\% confidential intervals. This result demonstrates that auditory reinforcement occurred in the same manner as that in Experiment 1 (Fig. 3). The subjects perceived the second sound stimulus to be approximately $1 \mathrm{~dB}$ louder than the first sound even when both sounds had the same SPL at an ISI of $0.5 \mathrm{~s}$. This result shows that auditory reinforcement is significant only immediately after comparison (ISI $=0.5 \mathrm{~s}$ ). In the comparison between Fig. 3 (base sound: $80 \mathrm{~dB}$ SPL) and Fig. 4 (base sound: $60 \mathrm{~dB}$ SPL), the auditory reinforcement levels are largest at an ISI of $0.5 \mathrm{~s}$ and have almost the same tendencies. However, auditory reinforcement is significant at ISIs from 0.5 to $2 \mathrm{~s}$ in Fig. 3, whereas it is only present at an ISI of $0.5 \mathrm{~s}$ in Fig. 4. This shows the effective duration of auditory reinforcement increases as the SPL of the first sound stimulus becomes greater.

\section{DISCUSSION}

In this study, the previous sound effect in the contralateral ear was derived from the experimental results. It was found that the auditory sensitivity to loudness was significantly increased in the perception of a second sound perceived immediately after perceiving a previous sound in the contralateral ear, i.e., "auditory reinforcement" was observed. This phenomenon is different from "loudness enhancement," in which the loudness of a sound is not actually increased $[11,17]$. In the experiment on loudness enhancement, three sound stimuli were used, and the stimuli affected each other in a very complex fashion, as mentioned in Sect. 1. As a result, loudness enhancement was mistaken to be an actual increase in loudness sensitivity. In contrast, two pure tones were used in the experiments in this study, and the previous sound effect was evaluated on the basis of response probability transitions in paired comparisons without using a third stimulus. This method revealed that the loudness of a sound is essentially increased by the previous sound effect in the contralateral ear. Also, the duration of the second sound $(5 \mathrm{~s})$ in this study was set longer than those of previous studies. Auditory adaptation might have occurred slightly when a subject was hearing the experimental stimuli. If the adaptation occurred, the loudness of the first sound decreased generally while hearing the first sound. After hearing the first sound, the auditory sensitivity was recovered in the silent duration (ISI). Therefore, the subject might feel the second sound relatively louder than the first sound by comparing between the end of the first sound, in which loudness was decreased by the adaptation, and the beginning of the second sound, in which loudness was recovered from the adaptation. Then, the presentation order effect could be assumed to become larger as ISI increases because the recovery duration from the adaptation increased. However, the effect level decreased depending on the ISI. Thus, it is considered that the effect was not generated by the auditory adaptation but by the change in the sensitivity to the second sound induced by perceiving the first sound in the contralateral ear.

In addition, when the first sound at $80 \mathrm{~dB}$ SPL was presented in the contralateral ear, an approximately $50 \mathrm{~dB}$ SPL sound was leaked in the ipsilateral ear in this experimental system. The measured increasing loudness in the contralateral ear, therefore, included the effect of the first sound at $80 \mathrm{~dB}$ in the contralateral ear and the effect at $50 \mathrm{~dB}$ in the ipsilateral ear. The effect of the leaked sound at $50 \mathrm{~dB}$ SPL in the ipsilateral ear was considered to decrease the loudness of the second sound very slightly from the result in the ipsilateral ear. Hence, the actual effect induced by perceiving the first sound in the contralateral ear must be a little larger than the measured increased loudness level.

Also, from the experimental result, the properties of auditory reinforcement can be hypothesized as follows: auditory reinforcement is thought to be a temporal effect because such reinforcement is suppressed as ISI increases, and the effective duration of auditory reinforcement increases as the SPL of presented sound increases.

On the other hand, it was clarified that the previous sound effect in the ipsilateral ear has a feature of deteriorate the loudness of the second presented sound. This effect was generally weakened with increasing ISI up to $8 \mathrm{~s}$. It is believed that the deterioration of loudness after the first sound is associated with the previous sound effect 
reported previously [3-12]. In particular, the experimental conditions of loudness recalibration [8-12] were almost the same as those in our experiments. When the same SPL sounds were used as the first and second sounds in the recalibration experiment reported by Mapes-Riordan et al., [9], the level of deterioration was almost the same as our results.

Furthermore, from the experimental results and discussions described above, we consider the mechanism of the auditory reinforcement as follows: when the subject perceives a first sound in one ear, the auditory peripheral nervous system (only the same side of the ear) and central nervous system are stimulated. Immediately after perceiving the first sound, it is assumed that auditory sensitivity decreases in the peripheral nervous system depending on auditory fatigue, and it increases in the central nervous system. When the second sound is perceived in the contralateral ear, the opposite side peripheral system, where auditory sensitivity is not decreased yet, is stimulated and the central system, where the sensitivity is increased by the perception of the first sound, is stimulated. Thus, the second sound is perceived louder than the first sound owing to the previous sound effect. In the case of the ipsilateral ear, auditory sensitivity decreases in the peripheral system and increases in the central system with the perception of the first sound. Here, it is supposed that the deterioration level in the peripheral system is larger than the enhancement level in the central system. As a result, the second sound is perceived softer than the first sound.

\section{CONCLUSION}

In this study, paired comparison experiments were performed on the loudness of pure tones at various ISIs. In these experiments, the first sound of a comparison pair was presented to one ear, and then the second sound was presented in the contralateral or ipsilateral ear. From the experiments, the following results were obtained:

(1) Auditory sensitivity to loudness increased by approximately $1 \mathrm{~dB}$ in the contralateral ear immediately after hearing the sound at 80 or $60 \mathrm{~dB}$ SPL, i.e., "auditory reinforcement" occurred. This effect decreased as ISI was increased.

(2) Auditory reinforcement in the case of the base sound at $80 \mathrm{~dB}$ SPL lasted longer than that in the case of $60 \mathrm{~dB}$ SPL.

(3) Auditory sensitivity to loudness deteriorated by approximately $1 \mathrm{~dB}$ in the ipsilateral ear immediately after hearing a sound at $80 \mathrm{~dB}$ SPL. This effect decreased as ISI was increased.

In future studies, auditory reinforcement will be investigated in detail, including its frequency dependency and mechanism.

\section{ACKNOWLEDGMENTS}

The authors gratefully acknowledge partial supports to this work: a Grant-in-Aid for Young Scientists (B) (No. 15700145) and a Grant-in-Aid for Scientific Research (C) (2) (No. 15500127) from the Japanese Ministry of Education.

\section{REFERENCES}

[1] B. Scharf, "Sequential effects in loudness," Fechner Day 2001, pp. 1-6 (2001).

[2] B. C. J. Moore, An Introduction to the Psychology of Hearing, K. Ohgushi, Ed. (Seishinshobo, Tokyo, 1989), pp. 73-80.

[3] I. J. Hirsh and R. C. Bilger, "Auditory-threshold recovery after exposures to pure tones," J. Acoust. Soc. Am., 27, 1186-1194 (1955).

[4] I. J. Hirsh and W. D. Ward, "Recovery of the auditory threshold after strong acoustic stimulation," J. Acoust. Soc. Am., 27, 131-141 (1952).

[5] M. C. Botte, S. Charron and H. Bouayad, "Temporary threshold and loudness shifts: Frequency patterns and correlations," J. Acoust. Soc. Am., 93, 1524-1534 (1993).

[6] M. C. Botte and S. Mönikheim, "New data on the short-term effects of tone exposure," J. Acoust. Soc. Am., 95, 2598-2605 (1994).

[7] L. E. Marks, "Recalibrating" the auditory system: The perception of loudness," J. Exp. Psychol. Hum. Percept. Perform., 20, 382-396 (1994).

[8] Y. Arieh and L. E. Marks, "Recalibrating the auditory system: A speed-accuracy analysis of intensity perception," J. Exp. Psychol. Hum. Percept. Perform., 29, 523-536 (2003).

[9] D. Mapes-Riordan and W. A. Yost, "Loudness recalibration as a function of level," J. Acoust. Soc. Am., 106, 3506-3511 (1999).

[10] B. Nieder, S. Buus, M. Florentine and B. Scharf, "Effect of duration and level of the recalibration tone on the amount of loudness recalibration," J. Acoust. Soc. Am., 109, 2349 (2001).

[11] Y. Arieh and L. E. Marks, "Time course of loudness recalibration: Implications for loudness enhancement," $J$. Acoust. Soc. Am., 114, 1550-1556 (2003).

[12] B. Nieder, S. Buus, M. Florentine and B. Scharf, "Interactions between test- and inducer-tone durations in induced loudness reduction," J. Acoust. Soc. Am., 114, 2846-2855 (2003).

[13] R. Elmasian and R. Galambos, "Loudness enhancement: monaural, binaural, and dichotic," J. Acoust. Soc. Am., 58, 229-234 (1975).

[14] R. Elmasian, R. Galambos and A. Bernheim, "Loudness enhancement and decrement in four paradigms," J. Acoust. Soc. Am., 67, 601-607 (1980).

[15] F.-G. Zeng, "Loudness growth in forward masking," J. Acoust. Soc. Am., 92, 2363-2364 (1992).

[16] F.-G. Zeng, "Loudness growth in forward masking: Relation to intensity discrimination," J. Acoust. Soc. Am., 96, 2127-2132 (1994).

[17] B. Scharf, S. Buus and B. Nieder, "Loudness enhancement: Induced loudness reduction in disguise?," J. Acoust. Soc. Am., 112, 807-810 (2002).

[18] L. E. Marks, "Recalibrating the perception of loudness: Interaural transfer," J. Acoust. Soc. Am., 100, 473-480 (1996).

[19] J. Yoshida, H. Hasegawa and M. Kasuga, "Interstimulus interval dependence of the loudness difference limen obtained by taking into account the presentation order effect," Acoust. Sci. \& Tech., 25, 5, 311-317 (2004). 
[20] Y. Okamoto, "Analysis of all series data by maximum likelihood method," Kanazawa Univ.: Stud. Essays. Behav. Sci., 14, 63-71 (1994).

[21] Y. Okamoto "Analysis of all series data by maximum likelihood method: Study by simulation," Kanazawa Univ.: Stud. Essays. Behav. Sci., 15, 25-30 (1995).

[22] Y. Okamoto, "A number of sample in constant method," Kanazawa Univ.: Stud. Essays. Behav. Sci., 16, 23-29 (1996). 